\title{
Widespread occurrence of high-velocity upflows in solar active regions
}

\author{
S. L. Yardley ${ }^{1} \odot$, D. H. Brooks $s^{2,3}$, and D. Baker ${ }^{1} \odot$ \\ 1 University College London, Mullard Space Science Laboratory, Holmbury St. Mary, Dorking, Surrey RH5 6NT, UK \\ e-mail: stephanie.yardley@ucl.ac.uk \\ 2 College of Science, George Mason University, 4400 University Drive, Fairfax, VA 22030, USA \\ e-mail: dhbrooks.work@gmail.com \\ 3 Hinode Team, ISAS/JAXA, 3-1-1 Yoshinodai, Chuo-ku, Sagamihara, Kanagawa 252-5210, Japan
}

Received 19 April 2021 / Accepted 2 June 2021

\begin{abstract}
Aims. We performed a systematic study of 12 active regions (ARs) with a broad range of areas, magnetic fluxes, and associated solar activity in order to determine whether there are upflows present at the AR boundaries and, if these upflows exist, whether there is a high-speed asymmetric blue wing component present in them.

Methods. To identify the presence and locations of the AR upflows, we derive relative Doppler velocity maps by fitting a Gaussian function to Hinode/EIS Fe XII $192.394 \AA$ Aine profiles. To determine whether there is a high-speed asymmetric component present in the AR upflows, we fit a double Gaussian function to the Fe XII 192.394 ̊̊ mean spectrum that is computed in a region of interest situated in the AR upflows.

Results. Upflows are observed at both the eastern and western boundaries of all ARs in our sample, with average upflow velocities ranging between -5 and $-26 \mathrm{~km} \mathrm{~s}^{-1}$. A blue wing asymmetry is present in every line profile. The intensity ratio between the minor high-speed asymmetric Gaussian component compared to the main component is relatively small for the majority of regions; however, in a minority of cases (8/30) the ratios are large and range between 20 and $56 \%$.

Conclusions. These results suggest that upflows and the high-speed asymmetric blue wing component are a common feature of all ARs.
\end{abstract}

Key words. Sun: corona - Sun: UV radiation

\section{Introduction}

Identifying the origin of the slow speed $\left(\sim 500 \mathrm{~km} \mathrm{~s}^{-1}\right)$ solar wind and determining the structure and dynamics at its sources are amongst the most important goals of current research in heliophysics. With the recent launches of Parker Solar Probe (Müller et al. 2020) and Solar Orbiter (Fox et al. 2016) there is great interest in connecting observations made in situ in the solar wind with remote sensing measurements of the source regions. As part of that effort, characterising the physical properties of potential source regions is important.

Previous observations from the Hinode satellite (Kosugi et al. 2007) have shown that high temperature upflows are present at the boundaries of many solar active regions (ARs; Sakao et al. 2007; Harra et al. 2008; Del Zanna 2008; Tian et al. 2021). These upflows may be outflows if they have access to open magnetic fields that connect to the heliosphere (Sakao et al. 2007; Harra et al. 2008; Doschek et al. 2008; Baker et al. 2009), and elemental abundance measurements show that they have a coronal composition (Brooks \& Warren 2011). More precisely, the upflowing material may be a source of the slow solar wind. Brooks et al. (2015) found, by using full-Sun composition and Doppler maps along with the potential-field sourcesurface (PFSS) model, that the primary source contributing to the slow solar wind does appear to originate from the boundaries of ARs. Conversely, by using a global potential field model, Edwards et al. (2016) determined that AR upflows were not associated with open magnetic fields. However, the study by Edwards et al. (2016) only concentrated on analysing upflows present in a limited number of ARs. Furthermore, plasma that is confined along closed magnetic field could also contribute to the slow solar wind indirectly if it can escape along open magnetic field through reconnection. The Hinode Review Team (2019) describes the current state of AR outflow studies within the wider context of solar wind research. A more general review of solar wind studies is available in, for example, Abbo et al. (2016).

Typical bulk flow speeds measured in upflows by the Extreme-ultraviolet Imaging Spectrometer (EIS; Culhane et al. 2007) are $10-40 \mathrm{~km} \mathrm{~s}^{-1}$ with mass motions of $30-60 \mathrm{~km} \mathrm{~s}^{-1}$ (Brooks \& Warren 2011), but there is often a high-speed asymmetric component in the blue wing of the EIS spectral line profiles. This high-speed component is weak, with the intensity only amounting to around $10 \%$ of the total emission of the upflow (Brooks \& Warren 2012). This Letter focuses on key questions about these upflows and asymmetries.

Browsing the EIS database leaves one with the impression that the upflows are present in most ARs, but selection effects may play a role in this. For example, Hinode generally 
Table 1. NOAA ARs and EIS data used in this study.

\begin{tabular}{cccccc}
\hline \hline No. & $\begin{array}{c}\text { NOAA } \\
\text { AR }\end{array}$ & $\begin{array}{c}\text { Time } \\
(\mathrm{UT})\end{array}$ & $\begin{array}{c}X_{c} \\
(\operatorname{arcsec})\end{array}$ & $\begin{array}{c}Y_{c} \\
(\operatorname{arcsec})\end{array}$ & EIS file \\
\hline 1 & 11082 & 2010 Jun 19 01:57:44 & -306.4 & 439.3 & eis_11_20100619_014433 \\
2 & 11082 & 2010 Jun 21 01:46:37 & $\begin{array}{c}162.9 \\
405.2\end{array}$ & eis_11_20100621_011541 \\
3 & 11089 & 2010 Jul 23 15:03:07 & -363.4 & -453.6 & eis_11_20100723_143210 \\
4 & 11109 & 2010 Sep 29 23:51:36 & 361.5 & 261.5 & eis_11_20100929_223226 \\
5 & 11147 & 2011 Jan 21 14:10:50 & 26.6 & 476.5 & eis_11_20110121_133954 \\
6 & 11150 & 2011 Jan 31 11:25:19 & -470.0 & -250.6 & eis_11_20110131_102326 \\
7 & 11158 & 2011 Feb 12 15:32:13 & -248.4 & -211.8 & eis_11_20110212_143019 \\
8 & 11190 & 2011 Apr 11 12:00:42 & -492.6 & 281.0 & eis_11_20110411_105848 \\
9 & 11190 & 2011 Apr 15 01:17:19 & 218.1 & 304.4 & eis_11_20110415_001526 \\
10 & 11193 & 2011 Apr 19 13:32:20 & 36.3 & 363.5 & eis_11_20110419_123027 \\
11 & 11243 & 2011 Jul 2 03:38:08 & -299.0 & 216.6 & eis_11_20110702_030712 \\
12 & 11259 & 2011 Jul 25 09:36:09 & 224.7 & 323.4 & eis_11_20110725_090513 \\
13 & 11271 & 2011 Aug 21 12:25:42 & -50.8 & 150.8 & eis_11_20110821_105251 \\
14 & 11339 & 2011 Nov 8 19:14:27 & 88.1 & 258.4 & eis_11_20111108_181234 \\
15 & 11339 & 2011 Nov 10 11:33:19 & 406.0 & 266.8 & eis_11_20111110_100028 \\
\hline
\end{tabular}

Notes. The information in this table has been taken from Warren et al. (2012). In Cols. 4 and 5, $X_{c}$ and $Y_{c}$ correspond to the $X$ and $Y$ coordinates of the AR that have been differentially rotated to the centre of the EIS raster.

observes the largest, most active targets, and there is often a delay between the emergence of a new AR and its selection for observations. Furthermore, since observing the emergence phase is difficult, the formation of the upflows is often missed. If this is a quick process, it might well be the case that upflows exist for most of the lifetime of an AR (Démoulin et al. 2013; Zangrilli \& Poletto 2016; Baker et al. 2017; Harra et al. 2017). In contrast, we know that the evolution of the magnetic field affects how an AR decays and finally disperses (Baker et al. 2015; van Driel-Gesztelyi \& Green 2015), so at some point this process will affect the magnetic field at the AR boundary and potentially disrupt the upflows.

The high-speed component of the EIS line profiles has also received much attention but has only been observed in a relatively small number of case studies. Asymmetries are seen in and around the footpoints of coronal loops (Hara et al. 2008; Bryans et al. 2010; Tian et al. 2021) and have also been linked with chromospheric jets (De Pontieu et al. 2009). The magnitude of the asymmetries appears to depend on temperature and are often more pronounced in the upflows (Brooks \& Warren 2012), but many of these results are known from the analysis of single ARs. Since the asymmetries are weak and measurements are difficult, there may also be a tendency to analyse examples with a strong signal. This could potentially be misleading.

Here we perform the first systematic study of 12 ARs, three of which are observed for a second time later in their evolution, in order to answer two questions: (i) if upflows are present in all sizes of ARs, and (ii) if upflows are present, is there always a high-speed blue wing asymmetry in the high temperature line profiles.

The 12 ARs we study cover a range of activity levels and conditions. They have previously been analysed for different purposes by Warren et al. (2012), Brooks \& Warren (2016), and Viall \& Klimchuk (2017), so we already know a great deal about them. The total unsigned magnetic flux spans a factor of $\sim 7\left(4.08 \times 10^{21}-2.73 \times 10^{22} \mathrm{Mx}\right)$, and the AR area varies by a factor of $\sim 5\left(2.87 \times 10^{19}-1.48 \times 10^{20} \mathrm{~cm}^{2}\right)$. The emission measure distributions in the cores of the ARs are generally strongly peaked near $4 \mathrm{MK}$, with a tendency to be steeper for the regions of stronger magnetic flux. Mass motions in the AR core loops are typically modest $\left(\sim 17 \mathrm{~km} \mathrm{~s}^{-1}\right)$. There is evidence of widespread cooling from high temperatures in all these regions (Viall \& Klimchuk 2017). Only one of the ARs is in close proximity to a coronal hole; therefore, there is little possibility of interactions between the AR boundaries and neighbouring coronal holes. PFSS extrapolations of the regions, however, show that there is open magnetic field present at one or both of the AR boundaries for seven out of the twelve ARs. These open field regions are located close to the areas of the upflows we analyse. This suggests that for these regions the upflows are indeed outflows; however, this requires further investigation.

\section{Observations and methods}

Table 1 gives information on the ARs; their National Oceanic and Atmospheric Administration (NOAA) number, and disk location, along with the EIS observations we used for this analysis. The EIS data were processed using the standard eis_prep procedure available in SolarSoft. This routine accounts for the CCD dark current and pedestal, and it handles dusty, hot, and warm pixels and cosmic rays. For this study, however, we did not apply the photometric calibration. Previous work has shown that applying the calibration modifies the spectral line width (Brooks \& Warren 2016; Testa et al. 2016), and since this is a crucial parameter for studying the shape of the line profile, we decided to use the raw uncalibrated spectra.

EIS observes two wavelength bands, from 171-212 $\AA$ and from $245-291 \AA$. The spectral resolution of the instrument is $22 \mathrm{~m} \AA$. The datasets listed in Table 1 were obtained in a variety of observing modes, using both the $1^{\prime \prime}$ and $2^{\prime \prime}$ slits and exposure times of 30-60 s. A field-of-view of 100-360" was scanned in the solar-X direction while a slit height (in solar-Y) of 240-512" was used. Specific details for each dataset were provided by Brooks \& Warren (2016).

We used the Fe XII $192.394 \AA$ spectral line for this analysis. This clean and unblended line is the second strongest in the EIS AR spectra (Young et al. 2007; Brown et al. 2008), and is present in all our datasets. The line is formed at $1.6 \mathrm{MK}$ in equilibrium conditions, and this is a good representative temperature 

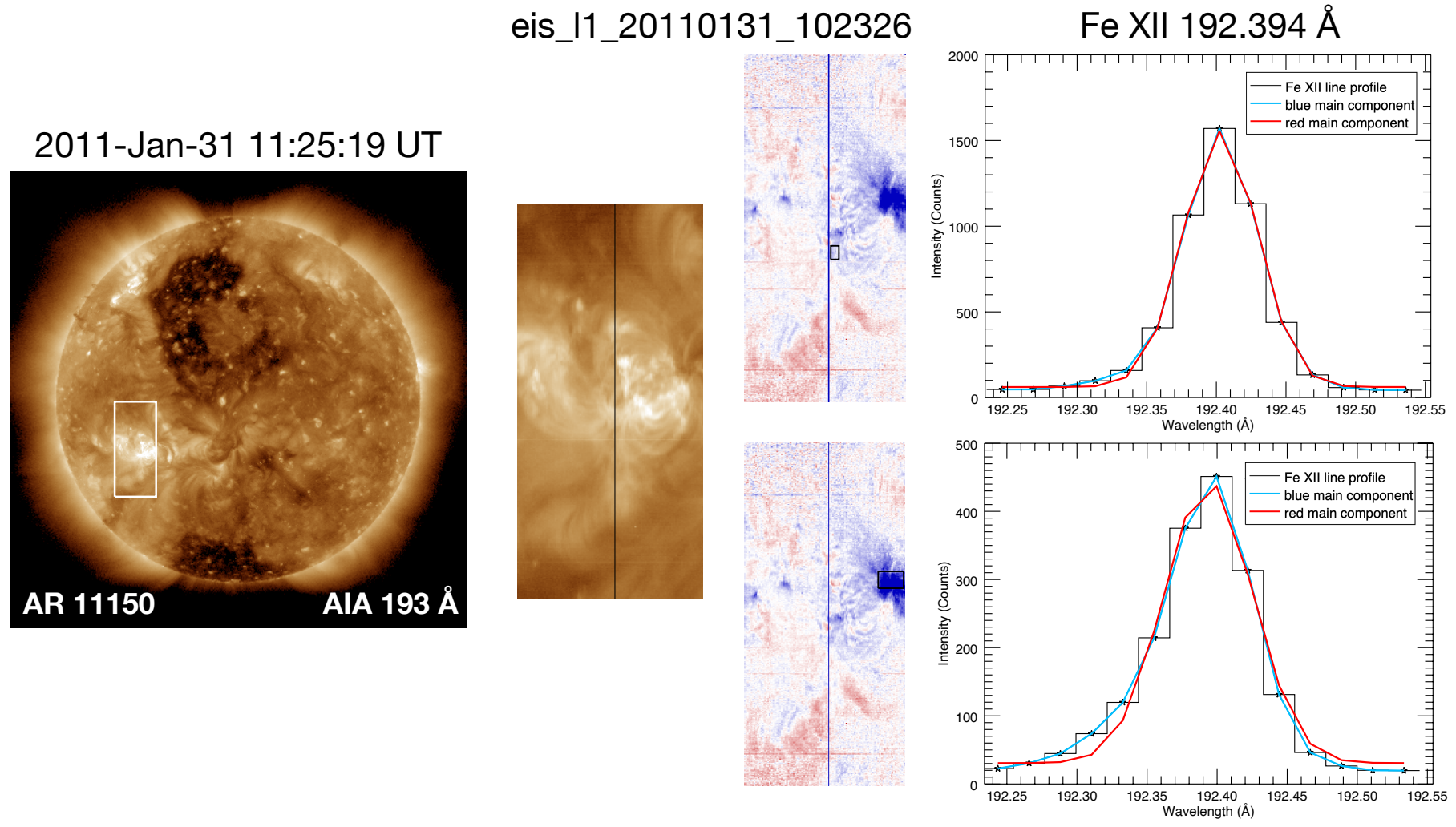

Fig. 1. Blue wing asymmetries for the eastern and western upflows of NOAA AR 11150 (No. 6 in Table 1). Left panel: $193 \AA$ image taken by the Solar Dynamics Observatory/Atmospheric Imaging Assembly (SDO/AIA), with the white box corresponding to the EIS field-of-view. Middle panel: EIS Fe XII $192.394 \AA$ image and Doppler velocity maps. Final panel: mean spectrum of the pixels highlighted by the black boxes in the EIS scans (middle panel), fitted with a single Gaussian.

for measuring upflows based on previous studies. To derive relative Doppler velocities from the Fe XII 192.394 $\AA$ line, we first removed the orbital drift of the EIS spectrum on the CCD using the neural network model of Kamio et al. (2010). This method uses instrument temperature information to model the drift of the Fe XII $195.119 \AA$ line as the satellite moves around its orbit. Since the Fe XII 192.394 $\AA$ and Fe XII 195.119 $\AA$ lines are very close in wavelength, the orbital correction for one should be valid for the other. In some cases we also applied an additional correction to account for any residual orbital variation that was not removed (see e.g., Brooks et al. 2020). To create relative Doppler velocity maps, we fitted a Gaussian function to the Fe XII 192.394 $\AA$ spectrum across each of the scans. These maps allowed us to easily identify upflow locations and determine whether they exist in every AR.

There are multiple methods that can be used to analyse the high-speed asymmetric component, such as a double Gaussian fit (Peter 2010; Bryans et al. 2010; Brooks \& Warren 2012), blue-red $(B-R)$ asymmetry analysis (De Pontieu et al. 2009; Tian et al. 2011), and the $B-R$ guided double Gaussian fit (De Pontieu \& McIntosh 2010; Tian et al. 2011). In this study, to assess whether a high-speed asymmetric component is present in the Fe XII 192.394 $\AA$ line profile, we used a double Gaussian function. We first selected a region of interest in the upflow and calculated the mean spectrum. We then fitted a single Gaussian function and used it to construct a double Gaussian template. The minor high-speed component was assumed to have $10 \%$ of the intensity of the main component, and both Gaussians were assumed to have the same width. The centroid of the minor component was initially placed in the blue wing with a
Doppler shift of $125 \mathrm{~km} \mathrm{~s}^{-1}$. This value is based on the results from Brooks \& Warren (2012); of course, the fit itself determines the actual Doppler shift. The assumption that the two components have the same width may not perfectly capture the smooth shape of the asymmetry, but it provides a consistent way of quantifying, for example, the area of the minor component compared to the main component across all the different ARs.

\section{Results}

Figures 1 and 2 show examples of the AR upflows and the asymmetric blue wing component present in the EIS Fe XII 192.394 A spectral line for ARs 11150 and 11193 (No. 6 and 10 in Table 1). The middle panels of both figures show the EIS Fe XII image along with the Doppler velocity maps of the ARs that have been derived using the methods outlined in Sect. 2. The Doppler velocity maps show that there are upflows (blue shifts) present at the eastern and western boundaries of both ARs. The upflows are particularly strong at the western boundary of AR 11150, whereas the strongest upflow for AR 11193 is located at the eastern boundary. We found these upflows to be present at the eastern and western boundaries in the Doppler maps for each AR in our sample.

The mean spectral profile is given in the final panels of Figs. 1 and 2 for the pixels indicated by the black boxes in the Doppler maps (middle panel). Each spectral profile shows a blue wing asymmetry. This high-speed asymmetric component is relatively weak, although its strength varies depending upon the upflow and selected region. We found these blue wing 

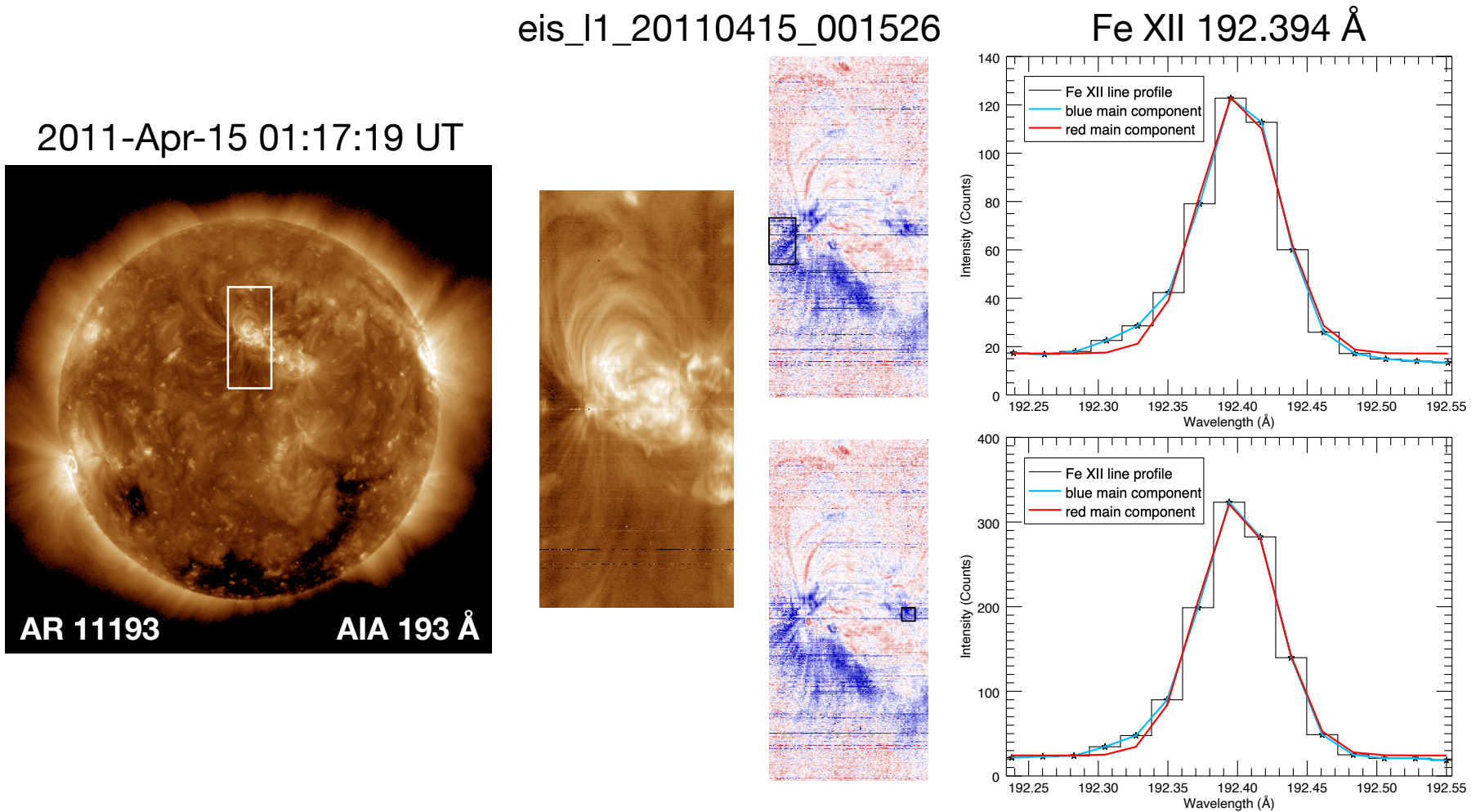

Fig. 2. Same as Fig. 1 but for NOAA AR 11193 (No. 10 in Table 1).

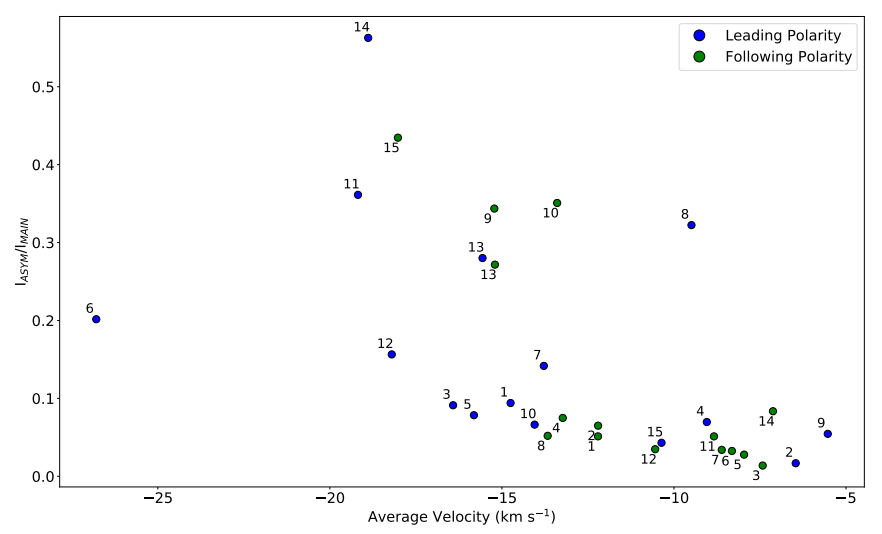

Fig. 3. Intensity ratio of the asymmetric and main component Gaussians as a function of the average velocities calculated for the regions of interest located in the eastern and western upflows of each AR. The blue (green) circles represent the average velocities associated with the leading (following) polarity of the AR. The numbers correspond to the EIS scans of the ARs given in Table 1 .

asymmetries to be present in all of the line profiles constructed from the regions of interest located in the AR upflows.

Figure 3 shows the intensity ratio of the asymmetric and main component Gaussians as a function of the average velocity calculated for the regions of interest for the eastern and western upflows for each AR. The average velocities have been shifted by $-5 \mathrm{~km} \mathrm{~s}^{-1}$ to account for the formation temperature of the $\mathrm{Fe}$ XII line (see Fig. 6 of Peter \& Judge 1999).

The blue (green) points and corresponding labels represent the average velocities obtained for the leading (following) polarities of the AR observations given in Table 1. The actual and true errors in the average velocities are found to be $\pm 4.63 \mathrm{~km} \mathrm{~s}^{-1}$ and $\pm 4.88 \mathrm{~km} \mathrm{~s}^{-1}$ and were calculated by taking the standard

deviation of the average velocities with and without the additional residual orbital correction discussed in Sect. 2, respectively. Figure 3 shows that the average velocities for the region of interest in each AR upflow are negative and that the blue wing asymmetric component is present in all ARs. There is a moderately strong negative correlation (Pearson correlation coefficient of -0.58) between the intensity ratio of the minor asymmetric component compared to the main component and the associated average velocity, though these quantities are not always independent. The average velocities for the AR upflows were found to be in the range of -5 to $-26 \mathrm{~km} \mathrm{~s}^{-1}$. For the majority of the upflows the intensity of the minor high-speed component is relatively small (i.e., less than $20 \%$ of the main component); however, for eight of the upflows the intensity ratio ranges from 20 to $56 \%$. In these cases the asymmetric component is rather broad due to the assumptions that have been made regarding the double Gaussian template. We also found a moderate positive correlation between the total unsigned flux of the ARs and the intensity ratio (Pearson correlation coefficient of 0.49 ). The relative intensity varies widely depending upon the location of the upflow (i.e., whether we are considering the upflow situated at the boundary of the leading or following polarity) and the stage of evolution of the AR. Similarly, these variations are also observed for the intensity ratio as a function of average velocity, as shown in Fig. 3. Finally, no correlation was found between the total unsigned magnetic flux and the average upflow velocity.

\section{Summary and discussion}

We carried out a systematic study using the EIS observations of 12 ARs in order to determine: (i) whether upflows exist in all sizes of ARs and, if so, (ii) is there always a high-speed blue wing asymmetry present in the Fe XII $192.394 \AA$ line profile. The ARs in our sample spanned a wide range of total unsigned 
magnetic flux $\left(4.08 \times 10^{21}-2.73 \times 10^{22} \mathrm{Mx}\right)$ and areas $\left(2.87 \times 10^{19}\right.$ $\left.-1.48 \times 10^{21} \mathrm{~cm}^{2}\right)$. Three of our ARs were observed for a second time, roughly 2 to 4 days after the initial EIS observations, resulting in a sample of 15 AR measurements in our study. By creating Doppler velocity maps from the EIS scans of each AR it was possible to identify whether upflows were present in each $\mathrm{AR}$ and their locations. We found that these upflows were present at the eastern and western boundaries of all ARs in our sample. This suggests that upflows at the boundary are a common feature of ARs, irrespective of their size. The average velocities for the regions of interest located in the AR upflows were found to be between -5 and $-26 \mathrm{~km} \mathrm{~s}^{-1}$.

Baker et al. (2017) also reported large-scale upflows observed at the peripheries of both polarities in a sample of ten ARs with a similar flux range to the ARs in this work. They found that the majority of the long-term evolution of the upflows is attributed to projection effects that are dependent upon the AR's location on the disk. This effect is stronger in ARs associated with higher velocities. In this study we have analysed EIS scans of ARs that were taken at numerous longitudes ranging between $-492.6^{\prime \prime}$ and 406.0", and so projection effects will be present. In fact, there is a significant change in the upflows and intensity of the blue wing asymmetries after approximately 2 to 4 days for the three ARs (11082, 11190 , and 11339 in Table 1) that were observed by EIS more than once. However, in this particular study we are only interested in whether these upflows and asymmetries are present at the peripheries of ARs, not their evolution.

To determine whether a high-speed asymmetric component exists in the Fe XII $192.394 \AA$ spectral line, we used a double Gaussian function, which was based upon the mean spectrum calculated for a region of interest located in the upflows. We then measured the intensity of the minor asymmetric component relative to the main component for all ARs. While we acknowledge that in some cases, by using a double Gaussian fit with two components, the width does not entirely capture the shape of the blue wing asymmetry, the fit provides a way to consistently compare the area of the minor and main components. Furthermore, comparisons with artificial line profiles support this analysis strategy (Tian et al. 2011). Alternative methods would be to use an asymmetric Gaussian fit where the degree of asymmetry comes from the difference in the widths between the Gaussians that fit the red and blue wings, or to follow the $B-R$ asymmetry analysis introduced by De Pontieu et al. (2009). Differences in methodology, however, do not change our fundamental results because the blue wing asymmetries were discovered to be present in each AR. This indicates that these asymmetries are likely to be present in all ARs. For most of our ARs the intensity of the high-speed component compared to the main component is quite small, with an intensity ratio of up to $20 \%$. However, for eight upflows the ratio of intensities are between 20 and $56 \%$.

We also found indications of moderate negative (positive) correlations between the intensity ratio of the minor asymmetric and main components and the average upflow velocities (total unsigned magnetic flux). However, the upflow velocity can vary significantly depending upon the location of the upflow; therefore, projection effects are potentially important. This requires full EIS coverage of the ARs. Other considerations need to be taken into account, such as the size and locations of the selected regions of interest and the fact that sizeable asymmetric components will shift a single Gaussian to the blue wing and will give larger Doppler velocities.

Prior observations have suggested that both the upflows located at the boundaries of ARs and the high-speed component are likely to be sources of the slow solar wind. The fact that they are present in all ARs suggests that their contribution could be significant. Previous studies have tried to link these features to in situ measurements of the solar wind, and we are currently investigating their role in the production of solar energetic particles (SEPs). Plasma composition measurements are important for SEP connection studies (Brooks \& Yardley 2021), and further work on this topic will be the focus of missions such as Solar Orbiter and Parker Solar Probe. In fact, Harra et al. (2021) have already analysed a series of small type III bursts observed by Parker Solar Probe in order to identify the source of SEPs. They found that the most likely source of the radio storm was an extended blue-shifted outflow region located at the eastern boundary of an expanding AR.

Acknowledgements. SLY would like to thank NERC for funding via the SWIMMR Aviation Risk Modelling (SWARM) Project, grant number NE/V002899/1. The work of DHB was performed under contract to the Naval Research Laboratory and was funded by the NASA Hinode program. DB is funded under STFC consolidated grant number ST/S000240/1. Hinode is a Japanese mission developed and launched by ISAS/JAXA, with NAOJ as domestic partner and NASA and STFC (UK) as international partners. It is operated by these agencies in co-operation with ESA and NSC (Norway).

\section{References}

Abbo, L., Ofman, L., Antiochos, S. K., et al. 2016, Space Sci. Rev., 201, 55 Baker, D., van Driel-Gesztelyi, L., Mandrini, C. H., Démoulin, P., \& Murray, M. J. 2009, ApJ, 705, 926

Baker, D., Brooks, D. H., Démoulin, P., et al. 2015, ApJ, 802, 104

Baker, D., Janvier, M., Démoulin, P., \& Mand rini, C. H., 2017, Sol. Phys., 292, 46

Brooks, D. H., \& Warren, H. P. 2011, ApJ, 727, L13

Brooks, D. H., \& Warren, H. P. 2012, ApJ, 760, L5

Brooks, D. H., \& Warren, H. P. 2016, ApJ, 820, 63

Brooks, D. H., \& Yardley, S. L. 2021, Sci. Adv., 7, 11

Brooks, D. H., Ugarte-Urra, I., \& Warren, H. P. 2015, Nat. Commun., 6, 5947

Brooks, D. H., Winebarger, A. R., Savage, S., et al. 2020, ApJ, 894, 144

Brown, C. M., Feldman, U., Seely, J. F., Korendyke, C. M., \& Hara, H. 2008, ApJS, 176, 511

Bryans, P., Young, P. R., \& Doschek, G. A. 2010, ApJ, 715, 1012

Culhane, J. L., Harra, L. K., James, A. M., et al. 2007, Sol. Phys., 243, 19

Del Zanna, G. 2008, A\&A, 481, L49

Démoulin, P., Baker, D., Mandrini, C. H., \& van Driel-Gesztelyi, L. 2013, Sol. Phys., 283, 341

De Pontieu, B., \& McIntosh, S. W. 2010, ApJ, 722, 1013

De Pontieu, B., McIntosh, S. W., Hansteen, V. H., \& Schrijver, C. J. 2009, ApJ, 701, L1

Doschek, G. A., Warren, H. P., Mariska, J. T., et al. 2008, ApJ, 686, 1362

Edwards, S. J., Parnell, C. E., Harra, L. K., Culhane, J. L., \& Brooks, D. H. 2016, Sol. Phys., 291, 117

Fox, N. J., Velli, M. C., Bale, S. D., et al. 2016, Space Sci. Rev., 204, 7

Hara, H., Watanabe, T., Harra, L. K., et al. 2008, ApJ, 678, L67

Harra, L. K., Sakao, T., Mandrini, C. H., et al. 2008, ApJ, 676, L147

Harra, L. K., Ugarte-Urra, I., De Rosa, M., et al. 2017, PASJ, 69, 47

Harra, L., Brooks, D. H., Bale, S. D., et al. 2021, A\&A, 650, A7

Hinode Review Team (Al-Janabi, K., et al.) 2019, PASJ, 71, R1

Kamio, S., Hara, H., Watanabe, T., Fredvik, T., \& Hansteen, V. H. 2010, Sol. Phys., 266, 209

Kosugi, T., Matsuzaki, K., Sakao, T., et al. 2007, Sol. Phys., 243, 3

Müller, D., St. Cyr, O. C., Zouganelis, I., et al. 2020, A\&A, 642, A1

Peter, H. 2010, A\&A, 521, A51

Peter, H., \& Judge, P. G. 1999, ApJ, 522, 1148

Sakao, T., Kano, R., Narukage, N., et al. 2007, Science, 318, 1585

Testa, P., De Pontieu, B., \& Hansteen, V. 2016, ApJ, 827, 99

Tian, H., McIntosh, S. W., De Pontieu, B., et al. 2011, ApJ, 738, 18

Tian, H., Harra, L., Baker, D., Brooks, D. H., \& Xia, L. 2021, Sol. Phys., 296, 47

van Driel-Gesztelyi, L., \& Green, L. M. 2015, Liv. Rev. Sol. Phys., 12, 1

Viall, N. M., \& Klimchuk, J. A. 2017, ApJ, 842, 108

Warren, H. P., Winebarger, A. R., \& Brooks, D. H. 2012, ApJ, 759, 141

Young, P. R., Del Zanna, G., Mason, H. E., et al. 2007, PASJ, 59, S857

Zangrilli, L., \& Poletto, G. 2016, A\&A, 594, A40 